\title{
Impact of the Spectrum in the Annual Energy Production of Multijunction Solar Cells
}

\author{
Juan Villa and Antonio Martí
}

\begin{abstract}
In this work, we study the limiting efficiency of the annual energy production of multijunction solar cells both as independently connected (MJSC-IC) and as series connected (MJSCSC). Our calculations take into account the impact of hourly and daily variations of the solar spectrum and the geographical latitude. The results confirm the lower dependence with the solar spectrum of independently connected MJSCs and suggest that, in order to progress in practice toward higher annual energy production efficiencies, it could be advisable to develop a 5-junction MJSC-IC rather than to increase the number of solar cells from 5 to 6 under the MJSC-SC approach.
\end{abstract}

Index Terms-Air mass, annual energy efficiency, multi-junction solar cells, limiting efficiency.

\section{INTRODUCTION}

$\mathbf{M}$ ULTIJUNCTION solar cells (MJSCs) have the potential to better exploit the solar spectrum than single gap solar cells. Depending on how the cells are connected, MJSC can be

1) Series connected (MJSC-SC), when the anode (cathode) of each cell is connected to the cathode (anode) of the next. Due to this series connection between the cells, the full cell assembly exhibits only two external terminals.

2) Independently connected (MJSC-IC), when both the anode and the cathode of each cell are accessed independently.

The series connection under the MJSC-SC approach imposes a constraint by which the electric current flowing through all the solar cells has to be the same. This restriction makes the maximum power operating point of each cell to depend on each other. In the MJSC-IC approach, the independent connection between the cells eliminates this current constrain and causes the efficiency of the MJSC-IC to be higher than the efficiency of the MJSC-SC although at the cost of introducing the aforementioned terminals to each cell in the tandem.

In order to maximize the photovoltaic conversion efficiency of the MJSCs, the values of the gap of the solar cells have to be optimized. This optimization has already been carried out in the past by several authors [1]-[10]. However, we need to carry out this optimization process again due mainly to the fact that not all the values we need for our calculations are available and, often, they are not calculated for the most recent solar ASTM G173-03 spectrum standards. The results of this revised and completed optimization are provided in Section II.

On the other hand, MJSC-IC exhibits lower sensitivity to changes in the solar spectrum than MJSC-SC that impacts on the annual energy production of the cells. Furthermore, given the fact that optimized MJSC-IC is more efficient than MJSC-SC, it is worth calculating the amount of additional annual energy that it is possible to produce with photovoltaic systems based on MJSC-IC with respect to those based on MJSC-SC. Similar studies were only done for 500 sun and specific locations [11]. Our study is extended to MJSC-IC and for 1 sun and maximum concentration at the time it generalizes the theory to consider the air mass (AM) and the geographical latitude. This study is detailed in Section III.

Part of the results we shall show [see Figs. 2, 6, and 7(b)] (studies for 500 sun) was presented at the 43rd IEEE PVSC Conference [12]. In this work, we extend our study to other illumination conditions (one sun and maximum concentration) and extend the range of the plot in Fig. 6 from AM20 to AM38.2. Additionally, MJSCs are usually envisaged to be used in concentration photovoltaic (CPV) systems. However, due to the difficulty of the tracker to follow the sun at low solar altitudes [13], CPV systems are expected to collect energy for solar altitudes greater than 20-30 degrees that correspond in our model to relative AMs lower than 2.5 approximately. The impact of this limitation on the efficiency of the annual energy production of MJSC is calculated in Section IV.

\section{REVISION OF THE LIMITING EFFICIENCY OF MJSC-IC AND MJSC-SC}

Fig. 1 illustrates the system of MJSC-SC that, as anticipated in the introduction, exhibits two electric terminals for the whole system. $E_{n}$ indicates the energy bandgap of cell $n$. Solar cells are assumed to absorb all the light they receive that is characterized by photon energy above this gap. The MJSC-IC, not illustrated here, is similar but two independently electric terminals are extracted from each cell.

In order to calculate the limiting efficiency of the systems, each cell is assumed to have a selective reflector attached to its rear part that prevents radiative coupling between the cells. By doing so, photons emitted by cell $n$ through its rear side 


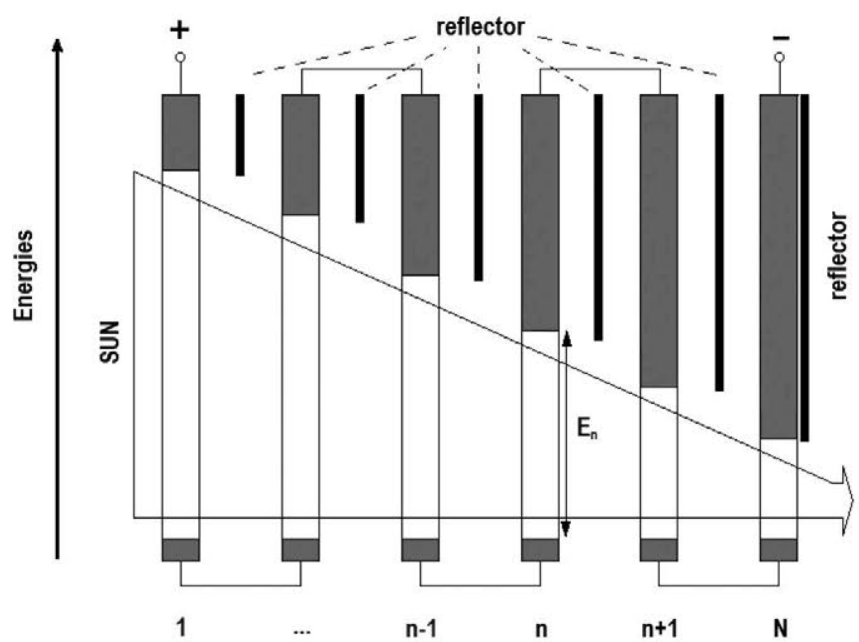

Fig. 1. Diagram of an MJSC-SC system. Selective mirrors have been placed between each solar cell to prevent radiative coupling between the cells. The last cell in the stack also contains a back reflector [12].

are reflected back toward the same cell and photons emitted by cell $n+1$ from its front side are reabsorbed by cell $n+1$. This approach is known to lead to the maximum efficiency. The theory related to the calculations of the efficiency limit of these systems was described in [1] and will not be repeated here. The calculations are based on the detailed balance theory formulated by Shockley-Queisser [14].

The spectrum used in the calculations is this work is the ASTM G173-03 reference spectrum. When different values of the relative air mass (or simply $\mathrm{AM}$ ) are needed, the spectrum is calculated making use of SMARTS2 v.2.9.2 simulation software [15] by keeping constant all the parameters characterizing ASTM G173-03 (humidity, aerosol, etc.) but changing the value of AM. For reference we shall mention that the integrated (by trapezoids) power for the AM1.5 direct normal+circumsolar irradiance conditions results in $901 \mathrm{~W} / \mathrm{m}^{2}$ and, for AM1.5 global, results in $1013 \mathrm{~W} / \mathrm{m}^{2}$ (which is the spectrum used when we will later refer ourselves to the 1 sun case).

Fig. 2 details the limiting efficiencies calculated for this spectrum, for the cells assumed at $300 \mathrm{~K}$, up to a system consisting of 6 cells as well as for the ideal case of a system of infinite cells for the cases of 1 sun, 500 sun, and maximum concentration (46 050 sun). The bandgaps of the cells have been optimized for each case and their values are represented in Figs. 3-5. The bars indicate the tolerance in the value of the optimum gap to preserve the maximum efficiency within $1 \%$ from the maximum when the value of the gap is modified. Finally, as an example to illustrate the results, Table I provides the optimum gaps of 6-MJSC both series connected and independently connected for the three illumination conditions.

As anticipated, MJSC-IC turns out to be more efficient than MJSC-SC. The relative difference between the limiting efficiencies of the MJSC-IC and those of the MJSC-SC for maximum concentration varies from $0.1 \%$ to $2.3 \%$. For a concentration of 500 sun, this relative difference in efficiency varies between $0.4 \%$ and $1.5 \%$. For a concentration of 1 sun, the relative difference changes from $0.6 \%$ to $1.6 \%$. Besides, a comparison between the limiting efficiencies calculated previously

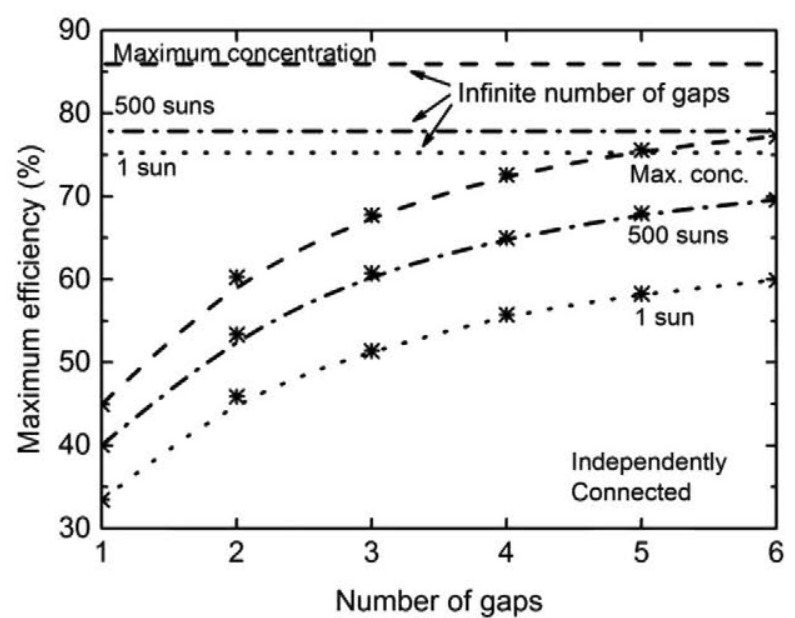

(a)

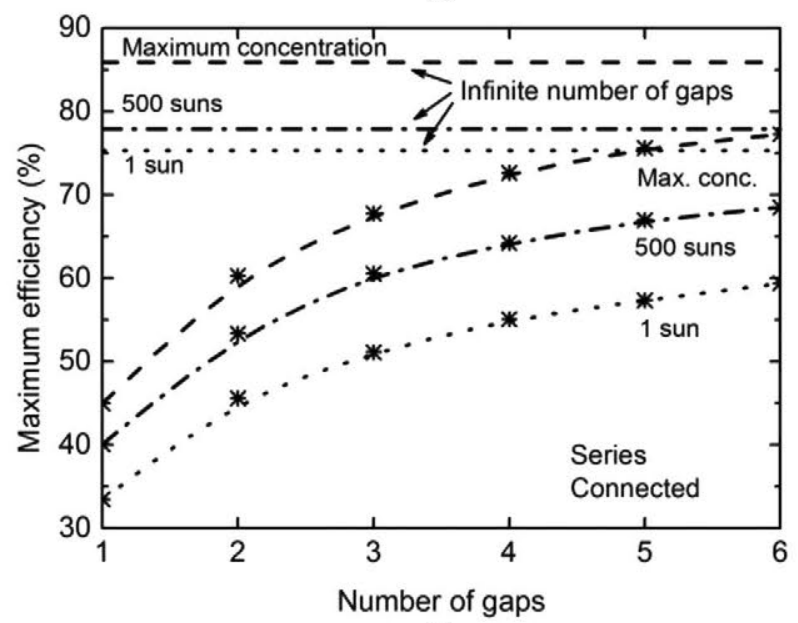

(b)

Fig. 2. Maximum efficiency as a function of the number of solar cells: (a) for independently connected cells and (b) for series-connected cells in the tandem system [12].

with the now obsolete spectra and the revised ones show, for example, that the revised limiting efficiency for four MJSC-SC results in $55.1 \%$ at 1 sun (while the previous one was $54.0 \%$ ), $64.2 \%$ for 500 sun (versus $63.6 \%$ ), and $72.2 \%$ for maximum concentration (versus $71.0 \%$ ). The limiting efficiency for single gap solar cells at 1 sun has also been calculated by Green [10] for the new ASTM G173-03 standard obtaining two maximum values: $33.8 \%$ for a bandgap of $1.34 \mathrm{eV}$ and $33.6 \%$ for $1.15 \mathrm{eV}$. Our limiting efficiencies for both bandgaps result in $33.5 \%$ in both cases.

The increase in the photovoltaic conversion efficiency saturates as the number of solar cells in the tandem increases. In fact, the limiting efficiency for both types of MJSCs becomes the same when an infinite number of solar cells is considered, as previously demonstrated in [16] and [17]. For our updated calculations, these limiting efficiencies result in: $85.9 \%$ for maximum concentration, $77.9 \%$ for a concentration of 500 sun, and $75.3 \%$ for 1 sun.

Another advantage of the MJSC-IC versus MJSC-SC is revealed by the tolerance of the optimum gaps to achieve the maximum efficiency which is higher for the IC case. In addi- 


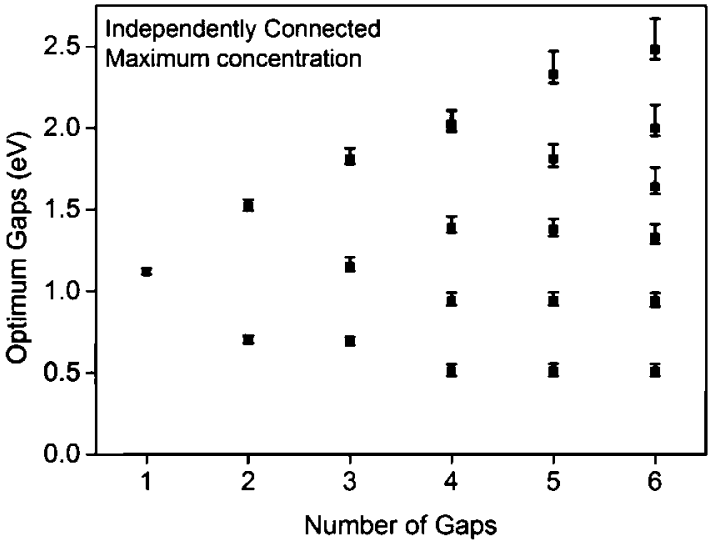

(a)

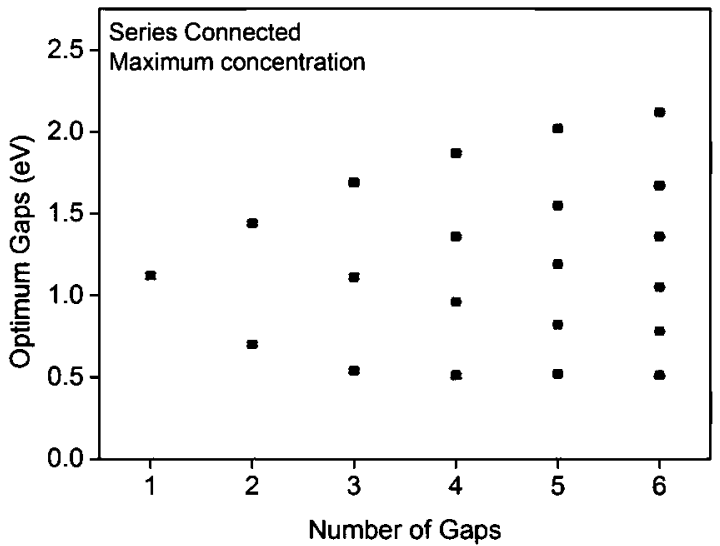

(b)

Fig. 3. Optimum gaps as a function of the number of cells in the stack for AM1.5 direct normal irradiance and maximum concentration: (a) MJSC-IC; and (b) MJSC-SC.

tion, the tolerance of the optimum bandgaps to changes in the solar spectrum is also higher in the IC than in the SC case.

\section{EFFICIENCY OF ANNUAL ENERGY PRODUCTION}

The annual energy production efficiency (AEE) is defined as the maximum photovoltaic energy produced by the cells in one year divided by the total energy received from the sun during the year. This efficiency is not to be confused with the efficiency of the cell, discussed in the previous sections, which is defined as the ratio between the maximum output power produced by the cell and the power of the solar irradiance input.

Since the solar spectrum changes along the daytime, in order to calculate the AEE it is necessary to know the efficiency of the solar cells as a function of the AM of the solar spectrum. This efficiency is plotted in Fig. 6. The value of the gaps of the cells is the optimum one calculated in Figs. 3-5, that is, the ones that are optimum for AM1.5.

$A M$ values can be related to the angular position of the sun in the sky. This AM value has been correlated with the terrestrial latitude and daytime through the equations given in [18].

In our calculations, the value of the AM has been approximated by

$$
\mathrm{AM}=\frac{1}{\cos \theta}
$$

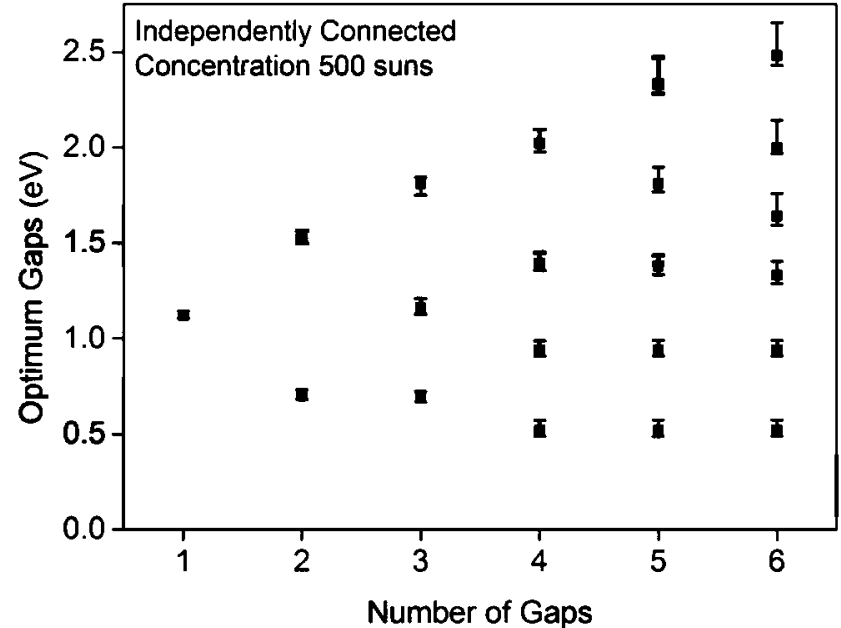

(a)

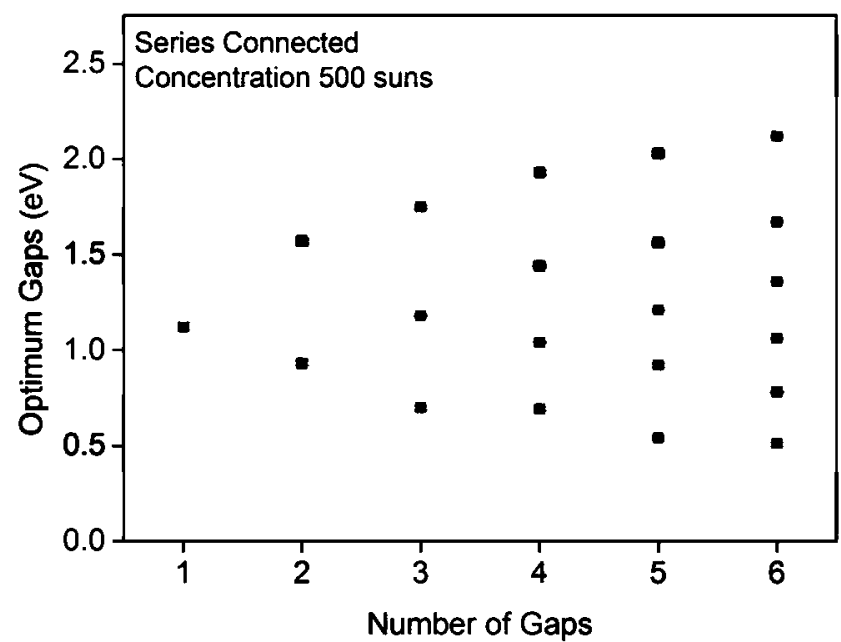

(b)

Fig. 4. Optimum gaps as a function of the number of cells in the stack for AM1.5 direct normal irradiance and 500 sun of concentration; (a) MJSC-IC; and (b) MJSC-SC.

where $\theta$ is the zenith angle of the sun. This equation assumes that the atmosphere is a flat horizontal layer and can be considered correct within 10\% for AM values lower than 15 [15], [19]-[21] which are the practical values used in this work.

AEE considers the whole amount of energy along the year reaching the MJSC taking into account the spectra for different relative AM coefficients. In practice, in our calculations, we have taken a maximum value of 20 for the AM coefficient where the solar irradiance is $133.4 \mathrm{~W} / \mathrm{m}^{2}$ for the Directo + Circumsolar spectrum and $155.1 \mathrm{~W} / \mathrm{m}^{2}$ for the Global spectrum. From AM15 to AM38.2, the solar irradiance has been considered negligible in terms of AEE.

With this knowledge, Fig. 7 plots the AEE for a terrestrial latitude of $\lambda=40^{\circ}$ and: (a) maximum concentration, (b) 500 sun, and (c) 1 sun.

MJSC-IC provides higher values of AEE than MJSC-SC. Moreover, in systems consisting of four solar cells, under illumination conditions of 500 sun of concentration and for $\lambda=40^{\circ}$ terrestrial latitude, it is more favorable to evolve from a system 


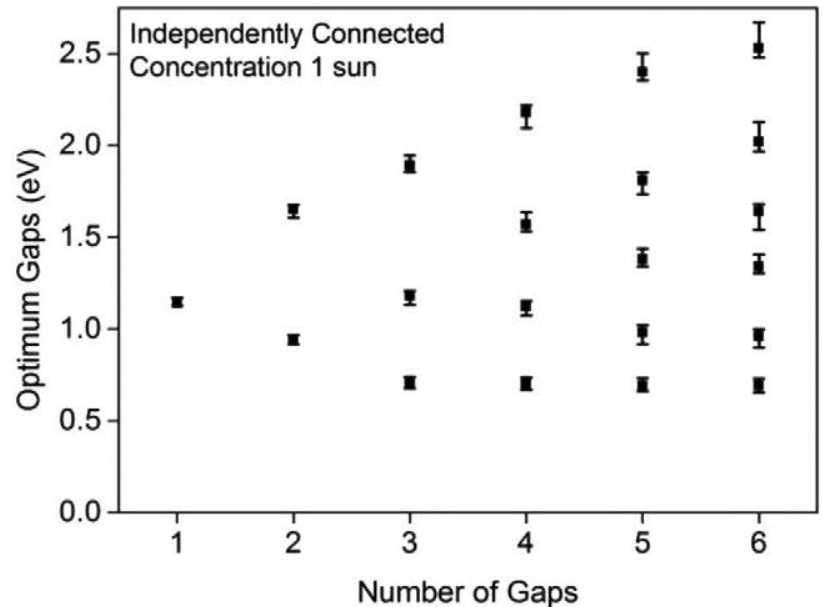

(a)

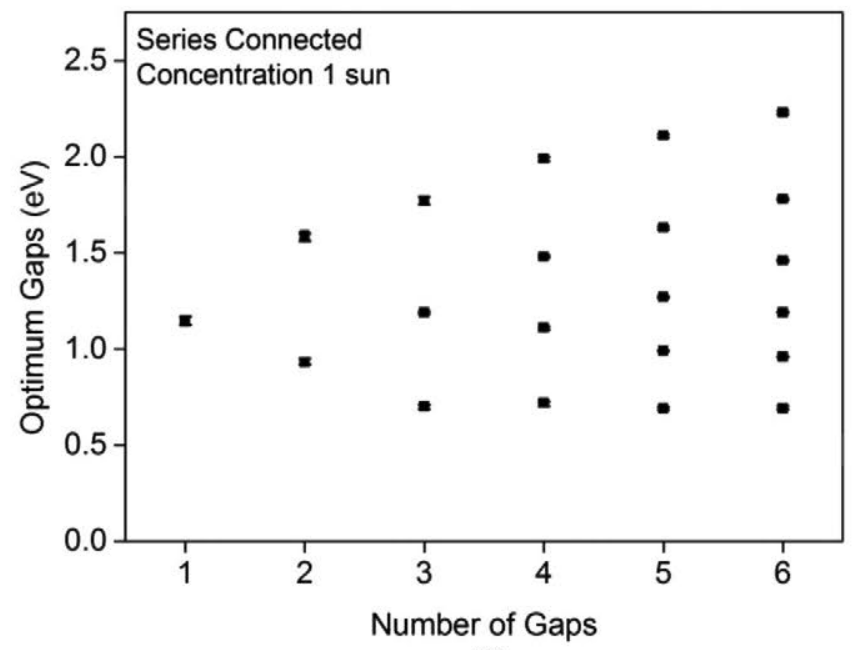

(b)

Fig. 5. Optimum gaps as a function of the number of cells in the stack for AM1.5 global irradiance (1 sun); (a) MJSC-IC; and (b) MJSC-SC.

TABLE I

OPTIMUM EFFICIENCY AND BANDGAPS FOR SIX CELLS

\begin{tabular}{lcccccc}
\hline \hline Description & \multicolumn{6}{c}{ Optimum gaps (eV) } \\
\cline { 2 - 7 } & E1 & E2 & E3 & E4 & E5 & E6 \\
\hline 1 sun series & 0.69 & 0.96 & 1.19 & 1.46 & 1.78 & 2.23 \\
500 sun series & 0.51 & 0.78 & 1.06 & 1.36 & 1.67 & 2.12 \\
500 sun indep. & 0.52 & 0.94 & 1.33 & 1.64 & 2.00 & 2.48 \\
Max. conc. series & 0.51 & 0.78 & 1.05 & 1.36 & 1.67 & 2.12 \\
Max. conc. indep. & 0.51 & 0.94 & 1.33 & 1.64 & 2.00 & 2.48 \\
\hline \hline
\end{tabular}

with series connection $(\mathrm{AEE}=60.3 \%)$ toward one with independently connected cells $(\mathrm{AEE}=64.9 \%)$ rather than evolving to an MJSC-SC of five solar cells ( $\mathrm{AEE}=61.6 \%)$. This advantage increases as the number of cells increases. Hence, for the case of MJSC-SC consisting of five solar cells (AEE $=61.6 \%$ ), it is more favorable to progress toward an MJSC-IC consisting also of five solar cells $(\mathrm{AEE}=67.7 \%)$ than toward an MJSC-SC of six solar cells ( $\mathrm{AEE}=63.2 \%$ ). Furthermore, this trend is also maintained for both under maximum concentration and 1 sun.

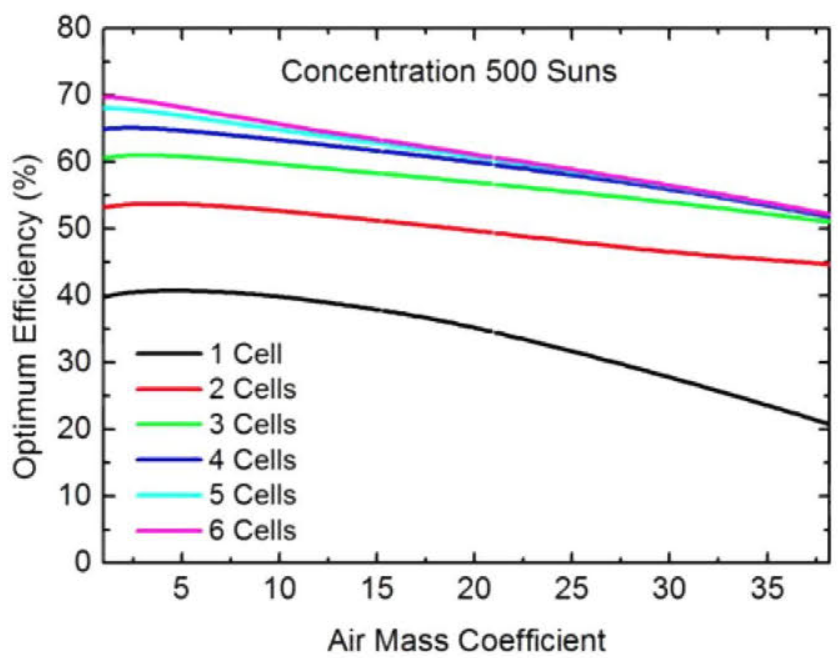

(a)

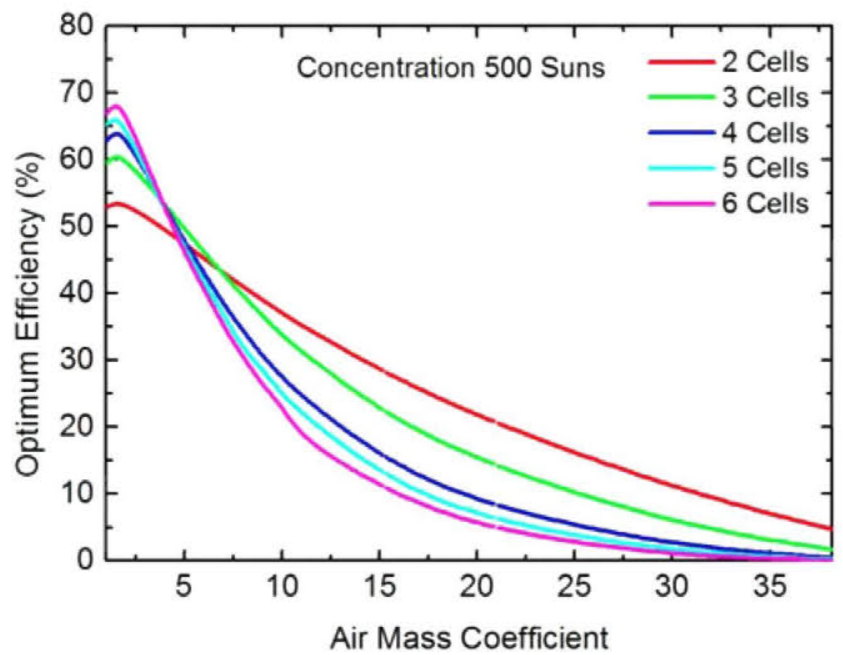

(b)

Fig. 6. Optimum efficiency for 500 sun of concentrated light as a function of the variation in the solar spectrum for different air mass coefficients (AM) for the case of: (a) MJSC-IC and (b) MJSC-SC (presented in [12] up to AM20).

\section{IMPACT OF LIMITED AM COLLECTION}

Calculations of the AEE of the MJSCs in the previous section assumed the ideal case in which the cells were able to collect all the sunlight along the daylight (that is, from the highest AM values-the solar spectrum at dawn or sunset times- to the lowest AM values corresponding to the solar zenith Fig. 8). In practical concentration systems, however, collecting light at high $\mathrm{AM}$ values is difficult due to the limitations of the tracking systems to point toward low altitude angles.

To study the impact of this limitation on the AEE, we will assume in this section that the capability of the system to collect sunlight is limited to AM values lower than a given AMX. In this respect, Fig. 9 shows a comparison between annual energy efficiencies of MJSC-SC and MJSC-IC taking into account different values of AMX for the case of a concentration of 500 sun. The results show that the difference between the AEE of MJSCSC and MJSC-IC increases as AMX increases. 


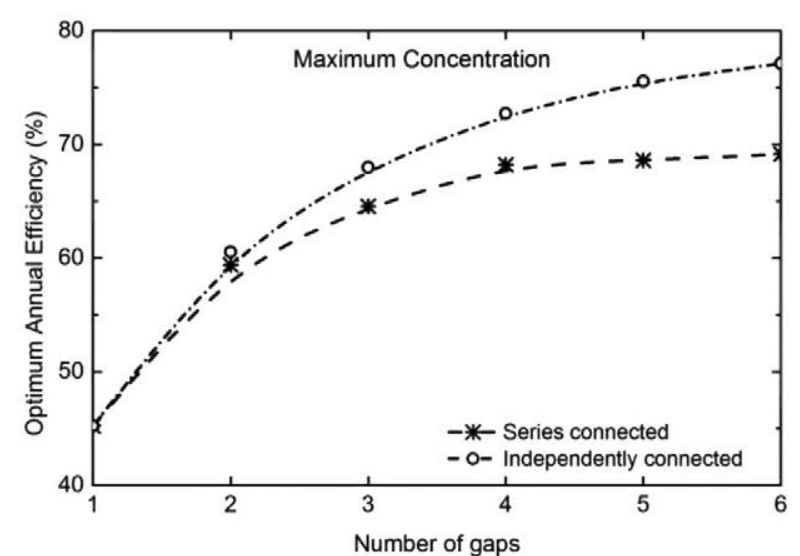

(a)

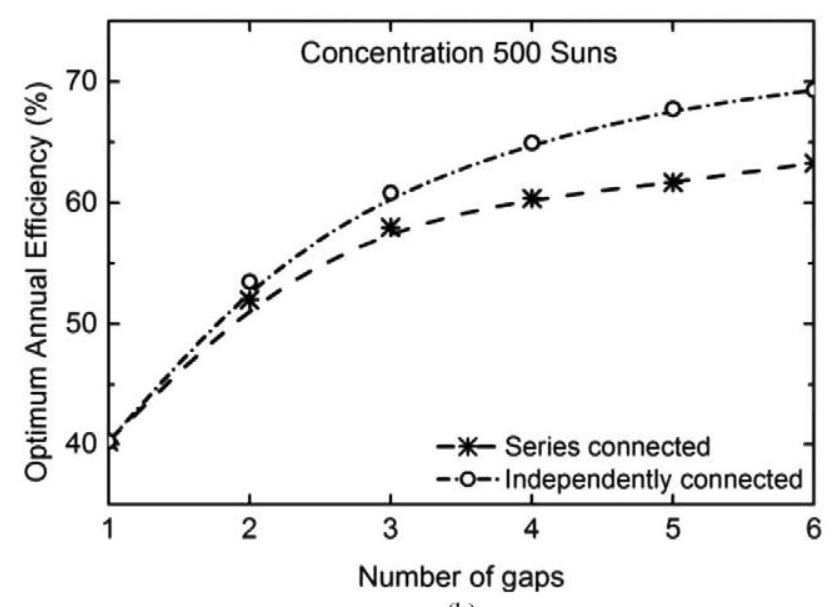

(b)

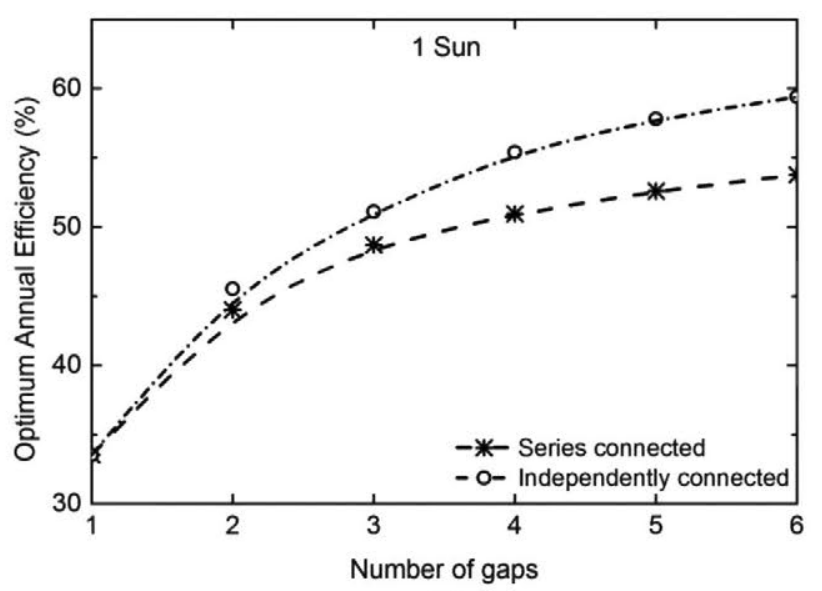

(c)

Fig. 7. Optimum annual efficiencies for stacked tandem systems with seriesconnected and independently connected solar cells for a terrestrial latitudes of $40^{\circ}$ and illumination conditions of: (a) maximum concentration, (b) concentration of 500 sun [12], and (c) 1 sun.

From these results, Fig. 10(a) plots the increase in AAE when considering going from an MJSC-SC system of three cells to an MJSC-IC of three cells and also to an MJSC-SC system of four cells as a function of AMX. As it can be seen, in this case, the MJSC-SC (three cells) to MJSC-SC (four cells) approach always leads to higher AEE increase. The situation changes

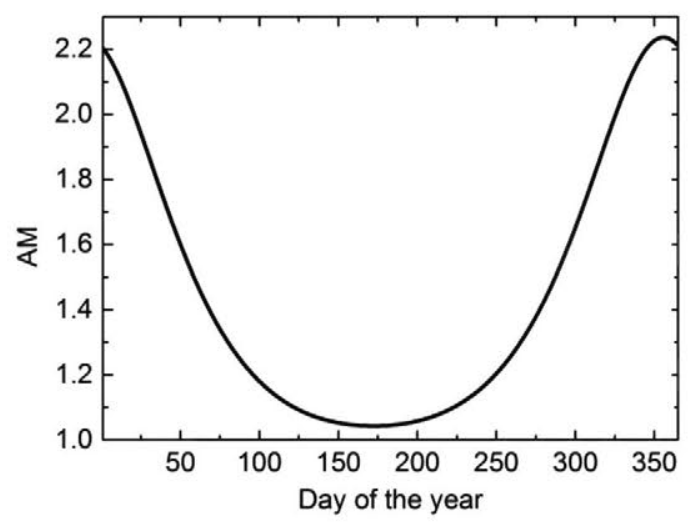

Fig. 8. AM at solar zenith for a terrestrial latitudes of $40^{\circ}$.

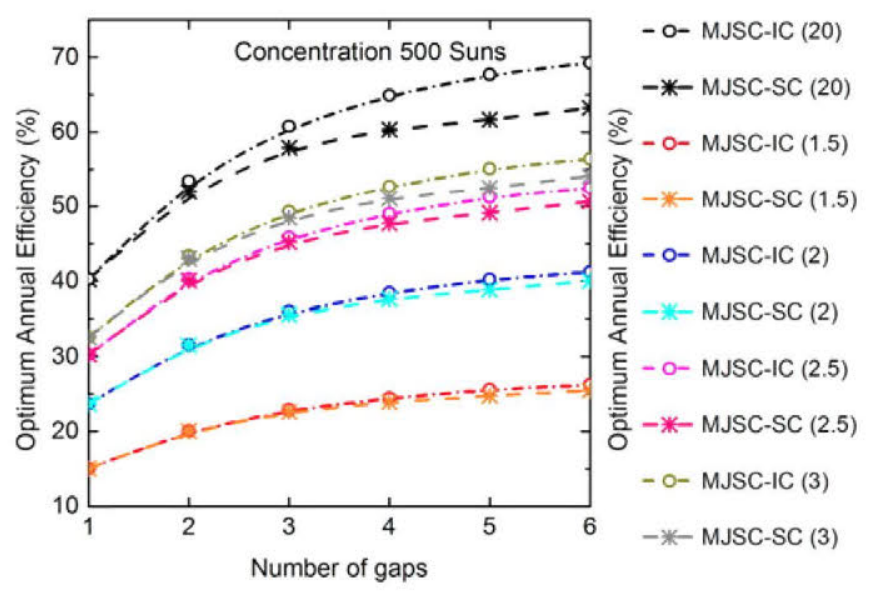

Fig. 9. Annual efficiencies for MJSC independently and series connected for different values of AMX indicated in brackets.

TABLE II

SOLAR TIME AT WHICH AIR MASS AM2.6 FOR TERRESTRIAL LATITUDES OF $\lambda=40^{\circ}$ IS OBTAINED FOR SEVERAL REPRESENTATIVE DATES ALONG THE YEAR

\begin{tabular}{ccc}
\hline \hline \multicolumn{3}{c}{ DATE } \\
\hline $03 / 21$ & 8:05 A.M. & 03:55 P.M. \\
$06 / 21$ & $6: 47$ A.M. & 05:13 P.M. \\
$09 / 21$ & $8: 03$ A.M. & 03:57 P.M. \\
$12 / 21$ & $10: 35$ A.M. & 01:25 P.M. \\
\hline \hline
\end{tabular}

when we compare going from an MJSC-SC system of four cells to an MJSC-SC system of five cells or to an MJSC-IC of also four cells [see Fig. 10(b)]. In this case, the MJSC-SC (four cells) to MJSC-IC (four cells) case results a better option for $\mathrm{AMX}>2.6$, that is, when the concentration system, with its tilt, is capable of collecting daily spectra characterized by AM higher than 2.6.

The AM2.6 spectrum corresponds to different hours for each day along the year. As an example, Table II indicates the solar hour at which the AM2.6 spectrum is obtained for several representative dates (solstices and equinoxes) for a terrestrial latitude $\lambda=40^{\circ}$. 


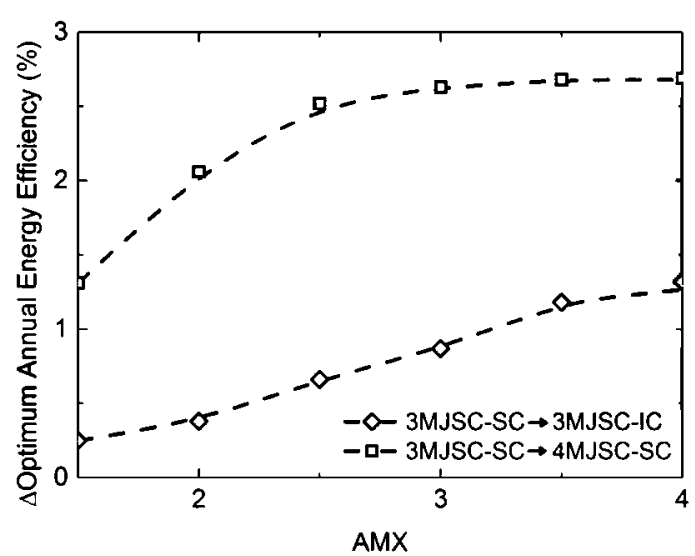

(a)

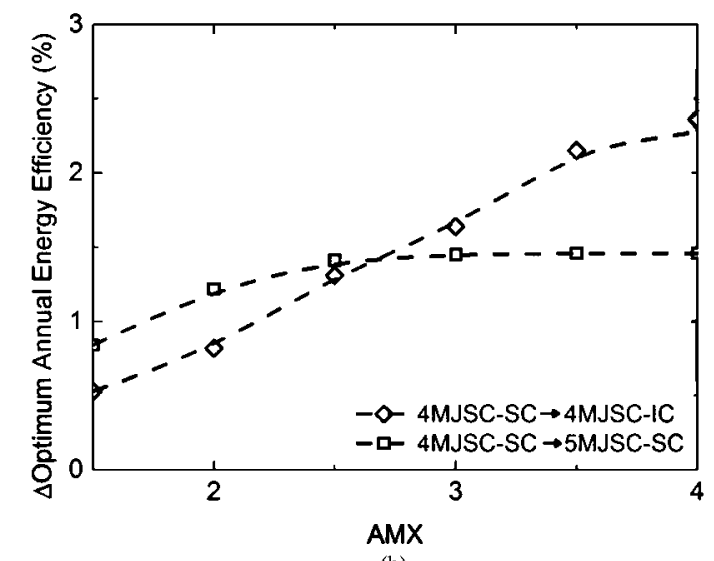

(b)

Fig. 10. Optimum annual efficiency changes as a function of AMX for a variation in the configuration of the MJSC (a) from 3MJSC-SC to 3MJSC-IC and from 3MJSC-SC to 4MJSC-SC; and (b) from 4MJSC-SC to 4MJSC-IC and from 4MJSC-SC to 5MJSC-SC.

\section{CONCLUSION}

The efficiency of photovoltaic conversion (for the new ASTM G173-03 reference spectra and different values for the relative AM) for tandem systems that are made up of 1-6 solar cells and an infinite number of cells, both series and independently connected, has been revised for the cases of 1 sun, 500 sun, and maximum concentrated light. As expected, the results show that MJSC-ICs have a greater photovoltaic conversion efficiency than the MJSC-SC, as long as the number of cells is finite, and the same limiting efficiency when the number of cells is infinite. On the other hand, the optimum value of the gaps of the MJSCIC exhibits a greater tolerance in order to achieve the maximum efficiency than for the MJSC-SC.

The optimum AEE of MJSC-SC and MJSC-IC consisting of several gaps has also been calculated for 1 sun, 500 sun, and maximum concentration. The results have been exemplified for a terrestrial latitude of $40^{\circ}$.

Due to the removal of the series connection constrain, as expected, MJSC-IC has shown higher AEE than MJSC-SC for the same number of cells. Furthermore, MJSC-IC of four gaps has revealed higher AEE than MJSC-SC of five gaps suggesting that going to an approach in which solar cells are independently connected could reveal itself more productive than increasing the number of cells in the system. In addition, our analysis of the AEE taking into account possible limitations in the collection of energy for high AM values has revealed that the advantage persists as long as the system is capable of collecting solar radiation at angles such that the AM is lower than 2.6.

\section{ACKNOWLEDGMENT}

Authors are grateful to Prof. G. Sala for suggesting studying the impact of AMX in the AEE.

\section{REFERENCES}

[1] A. Martí and G. L. Araújo, "Limiting efficiencies for photovoltaic energy conversion in multigap systems," Sol. Energy Mater. Sol. Cells, vol. 43, pp. 203-222, 1996.

[2] M. A. Green, "Third generation photovoltaics: Ultra-high conversion efficiency at low cost," Prog. Photovolt.: Res. Appl., vol. 9, pp. 123-135, 2001.

[3] S. Kurtz et al., "A comparison of theoretical and experimental efficiencies of concentrator solar cells," in Proc 4th Int. Conf. Sol. Concentrators Gener Electr. Hydrogen, El Escorial, Spain, 2007.

[4] S. Kurtz, D. Myers, W. E. McMahon, J. Geisz, and M. Steiner, "A comparison of theoretical efficiencies of multi-junction concentrator solar cells," Prog. Photovolt.: Res. Appl., vol. 16, pp. 537-546, 2008.

[5] A. De Vos, "Detailed balance limit of the efficiency of tandem solar cells," J. Phys. D: Appl. Phys., vol. 13, pp. 839-846, 1980.

[6] S. P. Bremner, M. Y. Levy, and C. B. Honsberg, "Analysis of tandem solar cell efficiencies under AM1.5G spectrum using a rapid flux calculation method," Prog. Photovolt.: Res. Appl., vol. 16, pp. 225-233, 2008.

[7] ASTM E891-87(1992), Tables for Terrestrial Direct Normal Solar Spectral Irradiance Tables for Air Mass 1.5 (Withdrawn 1999), ASTM E891-87, 1992.

[8] C. G. Abbot, "The Sun's energy-spectrum and temperature," Astrophys. J., vol. 34, pp. 197-208, 1911.

[9] Standard Tables for Reference Solar Spectral Irradiances: Direct Normal and Hemispherical on $37^{\circ}$ Tilted Surface, ASTM G173-03, 2008.

[10] M. A. Green, "Limiting photovoltaic efficiency under new ASTM International G173-based reference spectra," Prog. Photovolt.: Res. Appl., vol. 20, pp. 954-959, 2012.

[11] F. Dimroth et al., "Promises of advanced multi-junction solar cells for the use in CPV systems," in Proc. 2010 35th IEEE Photovolt. Spec. Conf., 2010, pp. 001231-001236.

[12] J. Villa and A. Marti, "Impact of the spectrum, location and interconnection between solar cells in the annual production of photovoltaic energies in photovoltaic concentration systems," in Proc. 2016 IEEE 43 rd Photovolt. Spec. Conf., 2016, pp. 0574-0576.

[13] P. Faine, S. R. Kurtz, C. Riordan, and J. M. Olson, "The influence of spectral solar irradiance variations on the performance of selected singlejunction and multijunction solar cells," Sol. Cells, vol. 31, pp. 259-278, 1991.

[14] W. Shockley and H. J. Queisser, "Detailed balance limit of efficiency of p-n junction solar cells," J. Appl. Phys., vol. 32, pp. 510-519, 1961.

[15] C. Gueymard, SMARTS2: A Simple Model of the Atmospheric Radiative Transfer of Sunshine: Algorithms and Performance Assessment. Cocoa, FL, USA: Florida Sol. Energy Center, 1995.

[16] A. S. Brown and M. A. Green, "Limiting efficiency for current-constrained two-terminal tandem cell stacks," Prog. Photovolt.: Res. Appl., vol. 10, pp. 299-307, 2002.

[17] I. Tobías and A. Luque, "Ideal efficiency of monolithic, series-connected multijunction solar cells," Prog. Photovolt.: Res. Appl., vol. 10, pp. 323$329,2002$.

[18] J. Greif, The European Solar Radiation Atlas, vol. 1. Paris, France: Presses des MINES, 2000.

[19] F. Kasten and A. T. Young, "Revised optical air mass tables and approximation formula," Appl. Opt., vol. 28, pp. 4735-4738, 1989.

[20] D. E. Green, "Magnitude corrections for atmospheric extinction," Int. Comet Quart., vol. 14, pp. 55-59, 1992.

[21] A. T. Young, "Air mass and refraction," Appl. Opt., vol. 33, pp. 1108-1110, 1994. 